\title{
SU(3) Breaking Effects in Hyperon Semi-Leptonic Decays and the extraction of $F$ and $D$
}

\author{
(submitted to Phys. Lett.) \\ Philip G. Ratcliffe \\ Dip. di Fisica, Univ. di Milano \\ via G. Celoria 16, 20133 Milano, Italy \\ E-mail:PGR@VAXMI.MI.INFN.IT
}

August 1995

\begin{abstract}
The analysis of hyperon semi-leptonic decay data is re-examined in the light of a recent suggestion that $\mathrm{SU}(3)$ symmetry breaking effects may be taken into account by applying a correction to the $F / D$ ratio obtained via naïve linear extrapolation in the hyperon masses. Comparison is made with the physically better motivated approach of applying so-called centre-ofmass corrections. This study (including all available data) reveals certain short-comings of the former of the above treatments, highlights some interesting aspects of this type of analysis and attempts to pinpoint the decay data that might usefully be improved. A tantalising result of the $\mathrm{SU}(3)$ breaking analysis performed here is that the magnitude of recoil correction required by the data corresponds closely to that required for the standard explanation of the reduction of $g_{A}$ from its $\mathrm{SU}(6)$ value of $5 / 3$. We also comment on other recent suggestions for taking into account the effects of SU(3) breaking. Finally a few remarks are made on the relevance for predicting the flavour non-singlet contribution to the proton $g_{1}$ and the Ellis-Jaffe sum rule.
\end{abstract}

PACS: 13.30.Ce, 13.88.+e, 11.30.Hv, 13.60.Hb

hep-ph/9509237 


\section{Introduction}

In the wake of the EMC measurement [1] of the spin-dependent proton structure function $g_{1}^{p}(x)$, much attention [2-7] has been focussed on the $F / D$ ratio extracted from hyperon semi-leptonic decay (HSD) data and used in predictions of the related Ellis-Jaffe sum rule [8]. In recent years the precision of experimental HSD data has improved considerably [9-12] with some parameters and rates now being measured to within an accuracy of a very few percent. Indeed, such is the present accuracy that an approach for applying corrections due to the breaking of $\mathrm{SU}(3)$ is now utterly indispensable. On the other hand, it has in the past even been suggested that the description in terms of the usual $F$ and $D$ parameters should be abandoned altogether [2].

With regard to the analysis of the above-mentioned EMC and more recent SMC and SLAC measurements of the nucleon spin structure functions $[13,14]$, a sizable shift in the $F / D$ ratio would remove the necessity for invoking a large strange-quark spin component of the proton [15] (referred to here as ES). The size of the shift from the naïve $\mathrm{SU}(3)$-based value depends strongly upon the framework used to describe the violating effects. It is important therefore to study the data with an eye to the sensitivity of the $F / D$ ratio to the assumptions made as to the effects of $\mathrm{SU}(3)$ breaking, which, in the past, have always been found to be at most of order $10 \%$, as might be expected a priori.

In this letter we shall examine the present data and, in particular, attempt to compare the various proposals for $\mathrm{SU}(3)$ breaking models and their effect on the interpretation of the data. In the following section we present the data used and, very briefly, the situation as regards the recurrent problem of discrepancies in the life-time and angular correlation measurements in neutron $\beta$-decay, and in section 3 we present the results of an $\mathrm{SU}(3)$ symmetric analysis as a reference point. In section 4 we examine and compare the effects of various possible $\mathrm{SU}(3)$-breaking scenarios. Thus, after establishing the nature of the problem, in section 5 we then examine recent proposals for dealing with $\mathrm{SU}(3)$ breaking. Finally, we present conclusions and some indications of the relevance to the proton-spin problem together with suggestions for further measurements aimed at better understanding hyperon semi-leptonic decays.

\section{Hyperon Semi-Leptonic Decay Data}

The HSD data considered here are shown in table I, which represents the useful knowledge presently available. A first important point is the widely discussed discrepancy between the neutron lifetime and the value of $g_{A} / g_{V}$ extracted from $\beta$-decay angular correlations [16]. In recent years both quantities have been subject to shifts and their experimental precision has significantly improved. The present value of the neutron lifetime is $887.0 \pm 2.0 \mathrm{~s}$ and $g_{A} / g_{V}$ (from angular cor- 
Table I: The hyperon semi-leptonic data used in this analysis [12], $g_{A} / g_{V}$ is as extracted from the angular correlations in the electron decay mode.

\begin{tabular}{|c|c|c|c|c|}
\hline \multirow{2}{*}{ Decay } & \multicolumn{2}{|c|}{ Rate $\left(10^{6} \mathrm{~s}^{-1}\right)$} & \multirow{2}{*}{$g_{A} / g_{V}$} & \multirow{2}{*}{$g_{A} / g_{V}$} \\
\hline & $\ell=e$ & $\ell=\mu$ & & \\
\hline$n \rightarrow p \ell \bar{\nu}$ & $1.1274 \pm 0.0025^{a}$ & & $1.2599 \pm 0.0025^{b}$ & $F+D$ \\
\hline$\Lambda^{0} \rightarrow p \ell \bar{\nu}$ & $3.161 \pm 0.058$ & $0.60 \pm 0.13$ & $0.718 \pm 0.015$ & $F+D / 3$ \\
\hline$\Sigma^{-} \rightarrow n \ell \bar{\nu}$ & $6.88 \pm 0.23$ & $3.04 \pm 0.27$ & $-0.340 \pm 0.017$ & $F-D$ \\
\hline$\Sigma^{-} \rightarrow \Lambda^{0} \ell \bar{\nu}$ & $0.387 \pm 0.018$ & & & $-\sqrt{\frac{2}{3}} D^{c}$ \\
\hline$\Sigma^{+} \rightarrow \Lambda^{0} \ell \nu$ & $0.250 \pm 0.063$ & & & $-\sqrt{\frac{2}{3}} D^{c}$ \\
\hline$\Xi^{-} \rightarrow \Lambda^{0} \ell \bar{\nu}$ & $3.35 \pm 0.37^{d}$ & $2.1 \pm 2.1^{e}$ & $0.25 \pm 0.05$ & $F-D / 3$ \\
\hline$\Xi^{-} \rightarrow \Sigma^{0} \ell \bar{\nu}$ & $0.53 \pm 0.10$ & & & $F+D$ \\
\hline
\end{tabular}

${ }^{a}$ Rate in $10^{-3} \mathrm{~s}^{-1}$.

${ }^{b}$ Taken from ref. [16].

${ }^{c} g_{V}=0$, absolute expression for $g_{A}$ given.

${ }^{d}$ Scale factor 2 included, following the PDG practice for discrepant data.

${ }^{e}$ Not used in fits.

relations) is $1.2599(25)$ [16], i.e., both are known independently to approximately $0.2 \%$. Using the $f t$ values from the eight super-allowed nuclear $\beta$-decay Fermi transitions, the relevant Cabibbo-Kobayashi-Maskawa (CKM) matrix element is $V_{u d}=0.9740(5)$ [17]; to be compared with the values: $V_{u d}=0.9795(20)$, from the neutron lifetime and $g_{A} / g_{V}$, and $V_{u d}=0.9758(4)$, from the so-called $K_{\ell 3}$ decays $\left(V_{u s}=0.2188(16)[18]\right)$.

Thus, there is no reason to assume any of the measurements to be more or less reliable, especially in view of the fact that the CKM unitarity violation for the two cases is of opposite sign. Moreover, the displacements from the central values are $<0.2 \%$, which nevertheless represents a much greater accuracy than is presently necessary for HSD analysis, or indeed for comparison with the Bjorken sum rule. Despite the interest in such a problem, it is beyond the scope of the present paper and it is reasonable for the purpose of this analysis to ignore the discrepancy; naturally though, its effects on the $\chi^{2}$ of fits obtained will be taken into account.

\section{$3 \quad \mathrm{SU}(3)$ Symmetric Analysis}

In order to have a clear idea of the problem within a phenomenological framework, let us make a first attempt at globally fitting the HSD data. Apart from a separation into lifetime and angular-correlation data, the distinction can also 
be made between strangeness conserving and changing decays. In table II we present the results of a series of three-parameter $\left(F, D\right.$ and $\left.V_{u d}\right)$ fits to different sub-classes of the HSD data alone; no value for $V_{u d}$ is imposed externally except for the final row, where we include the mean value obtained from the combined nuclear $f t$ analysis and $K_{\ell 3}$ decays just described. We shall also impose the constraint $V_{u d}^{2}+V_{u s}^{2}=1$ and thus neglect $V_{u b}\left(V_{u b}=0.0032 \pm 0.0009\right.$ [12]).

Table II: An SU(3) symmetric fit to the data.

\begin{tabular}{|c|c|c|c|c|c|}
\hline \multirow{2}{*}{ Data } & \multicolumn{3}{|c|}{ Parameters } & \multirow{2}{*}{$\chi^{2} /$ DoF } & $F / D$ \\
\cline { 2 - 4 } & $V_{u d}$ & $F$ & $D$ & & \\
\hline Rates & $0.9749 \pm 0.0004$ & $0.469 \pm 0.008$ & $0.797 \pm 0.008$ & 3.8 & 0.589 \\
\hline$g_{A} / g_{V}$ & $-^{a}$ & $0.460 \pm 0.008$ & $0.800 \pm 0.008$ & 0.8 & 0.576 \\
\hline$\Delta S=0$ & $0.9795 \pm 0.0020$ & $0.528 \pm 0.017$ & $0.732 \pm 0.017$ & $-{ }^{b}$ & 0.721 \\
\hline$|\Delta S|=1$ & $0.9742 \pm 0.0006$ & $0.448 \pm 0.009$ & $0.791 \pm 0.017$ & 0.8 & 0.567 \\
\hline All & $0.9750 \pm 0.0004$ & $0.465 \pm 0.006$ & $0.799 \pm 0.006$ & 3.0 & 0.582 \\
\hline All $+V_{u d}^{c}$ & $0.9751 \pm 0.0002$ & $0.465 \pm 0.006$ & $0.799 \pm 0.006$ & 2.7 & 0.583 \\
\hline
\end{tabular}

${ }^{a}$ Undetermined.

${ }^{b}$ Zero degrees of freedom.

${ }^{c} V_{u d}$ from nuclear $f t$ and $K_{\ell 3}$ analysis.

Two interesting points emerge from this fit: first of all, the value of $V_{u d}$ obtained solely from the hyperon data is consistent with that coming from the nuclear $f t$ analysis and $K_{\ell 3}$ data. Second, it should be pointed out that more than half the total $\chi^{2}$ (neglecting the discrepant neutron data contribution) comes from the $\Sigma^{ \pm} \rightarrow \Lambda^{0} \ell \nu$ data alone (where only the rates are accessible). This is interesting when considered together with the fact that these decays are unique in having no vector contribution and therefore, as we shall see later, have the rates most affected by the recoil corrections. Moreover, when fit together only with the other $|\Delta S|=0$ decays, they provide a value for $F / D$ close to the $\mathrm{SU}(6)$ prediction. This suggests that the problem may arise owing to the bulk of lesser affected data forcing a particularly poor value onto the one very sensitive point and not (as has been suggested [5]) that this experimental rate may be wrong; we shall return to this later.

Notice finally that, within the errors, the angular correlation data alone is well described by an SU(3) symmetric fit. Thus, one sees the futility of trying to extract any information on $\mathrm{SU}(3)$ breaking using these data alone and, as discussed later, the large errors arising in the ES analysis are partially explained.

Thus, to eliminate the effect of the neutron problem on the global $\chi^{2}$ we first extract a mean value for $V_{u d}$ from the nuclear $f t$ and $K_{\ell 3}$ data; using this value, we make a combined fit to the neutron rate and $g_{A} / g_{V}$. Then, in the absence 
of any indication as to where the problem may lie, we multiply the errors of the neutron lifetime, $g_{A}$ and mean $V_{u d}$ values by the $\sqrt{\chi^{2}}$ so obtained, and use these in all the following fits:

$$
\begin{aligned}
\operatorname{Rate}(n \rightarrow p \ell \bar{\nu}) & =(1.1274 \pm 0.0055) \times 10^{-3} s^{-1} \\
g_{A} / g_{V} & =1.2599 \pm 0.0055 \\
V_{u d} & =0.9752 \pm 0.0007
\end{aligned}
$$

in table III we display the $\mathrm{SU}(3)$ symmetric fit results using these values. It can

Table III: An SU(3) symmetric fit to the modified data including the external $V_{u d}$ from nuclear $f t$ and $K_{\ell 3}$ analysis (see text for details).

\begin{tabular}{|c|c|c|c|c|c|}
\hline \multirow{2}{*}{ Data } & \multicolumn{3}{|c|}{ Parameters } & \multirow{2}{*}{$\chi^{2} / \mathrm{DoF}$} & \multirow{2}{*}{$F / D$} \\
\cline { 2 - 4 } & $V_{u d}$ & $F$ & $D$ & & \\
\hline Rates & $0.9749 \pm 0.0003$ & $0.469 \pm 0.008$ & $0.796 \pm 0.009$ & 3.2 & 0.589 \\
\hline$g_{A} / g_{V}$ & $0.9752 \pm 0.0007$ & $0.460 \pm 0.008$ & $0.799 \pm 0.009$ & 0.8 & 0.576 \\
\hline$\Delta S=0$ & $0.9753 \pm 0.0007$ & $0.529 \pm 0.017$ & $0.735 \pm 0.017$ & 0.5 & 0.719 \\
\hline$|\Delta S|=1$ & $0.9747 \pm 0.0005$ & $0.452 \pm 0.009$ & $0.799 \pm 0.015$ & 0.8 & 0.566 \\
\hline All & $0.9749 \pm 0.0003$ & $0.465 \pm 0.006$ & $0.798 \pm 0.006$ & 2.3 & 0.582 \\
\hline
\end{tabular}

be seen that, having taken account of the neutron discrepancy, there is still a problem (stemming, as before, from the $\Sigma^{ \pm} \rightarrow \Lambda^{0} \ell \nu$ decay rate). Also worthy of remark is the fact that the $|\Delta S|=0$ and 1 data are separately well fit, suggestive of some correlated effect; we shall return to this later.

\section{$4 \quad \mathrm{SU}(3)$ breaking corrections}

The next step is to consider possible corrections to these processes. It has long been known that a realistic explanation of the renormalisation of the neutron $\beta$-decay $g_{A}$ can be provided in terms of relativistic corrections [19]. Such an approach has already been applied with success to the HSD data [4,20] and it has been noted that there might even be evidence that this accounts for $\mathrm{SU}(6)$ breaking [7].

One of the earliest analyses of this type [20] (referred to here as DHK) also attempted to include the effects of wave-function mismatch between the strange and $u, d$ quarks. However, the newer more precise data now strongly suggest that, at least as calculated there, such an effect is not present. On the other hand, since the data do seem to suggest some sort of correlated dependence, we shall also examine the effect of introducing an ad hoc correction for the $|\Delta S|=1$ decays. 
The DHK approach is to apply centre-of-mass (CoM) or recoil corrections to the axial coupling constant for the process $A \rightarrow B \ell \nu$ according to the following formula [20]:

$$
g_{A}=g_{A}^{\mathrm{SU}(3)}\left\{1-\frac{\left\langle p^{2}\right\rangle}{3 m_{A} m_{B}}\left[\frac{1}{4}+\frac{3 m_{B}}{8 m_{A}}+\frac{3 m_{A}}{8 m_{B}}\right]\right\},
$$

with a similar correction to the vector piece, which is entirely negligible (in accordance with the Ademollo-Gatto theorem [21]). The mean squared momentum, $\left\langle p^{2}\right\rangle$, is calculated by DHK using a bag model to be $0.43 \mathrm{GeV}^{2}$. In their analysis DHK use the ratio of the correction to that for the neutron (taken as a convenient reference value) since for the purposes of such a fit the important quantity is precisely this ratio. Thus, we begin the $\mathrm{SU}(3)$ breaking analysis using the DHK approach: the results are presented in table IV.

Table IV: An SU(3) breaking fit to the modified data including the external $V_{u d}$; only the approximate (DHK) CoM correction is applied (see text for details).

\begin{tabular}{|c|c|c|c|c|c|}
\hline \multirow{2}{*}{ Data } & \multicolumn{3}{|c|}{ Parameters } & \multirow{2}{*}{$\chi^{2} /$ DoF } & \multirow{2}{*}{$F / D$} \\
\cline { 2 - 4 } & $V_{u d}$ & $F$ & $D$ & & \\
\hline$\Delta S=0$ & $0.9753 \pm 0.0007$ & $0.481 \pm 0.018$ & $0.784 \pm 0.018$ & 0.5 & 0.613 \\
\hline$|\Delta S|=1$ & $0.9747 \pm 0.0005$ & $0.465 \pm 0.009$ & $0.825 \pm 0.015$ & 1.0 & 0.563 \\
\hline All & $0.9744 \pm 0.0003$ & $0.460 \pm 0.006$ & $0.806 \pm 0.006$ & 1.0 & 0.571 \\
\hline
\end{tabular}

The improvement is quite dramatic and is immediately seen to be essentially due to a sizable shift in the parameter values of the $\Delta S=0$ fit. The main effect is to increase (by about $6 \%$ ) the value of $D$ obtained from the $\Sigma^{ \pm} \rightarrow \Lambda^{0} \ell \nu$ decay rates (where recall $g_{V}=0$ ) and correspondingly reduce the value of $F$, thus bringing these decays into line with the rest (where the effect is more modest and acts to increase both $F$ and $D$ simultaneously). The overall $\chi^{2}$ is good and no single data point stands out as particularly poorly fit: the worst is $g_{A} / g_{V}$ for $\Xi^{-} \rightarrow \Lambda^{0} e \bar{\nu}$, which contributes 2.1 to the total $\chi^{2}$.

We note in passing that inclusion of the strange-quark wave-function mismatch correction, à la DHK, worsens the fits: without the CoM correction we obtain $\chi^{2}=5.6$ and with $\chi^{2}=2.6$ (for all data). One other observation of interest is that $\left\langle p^{2}\right\rangle=0.43$ actually corresponds to the best-fit value for this parameter, thus increasing the confidence in such an approach. Finally, note that the overall values of $F$ and $D$ have only been shifted by less than $1 \%$ and the ratio $F / D$ by less than $2 \%$.

Now we come to the possibility of a link with the $\mathrm{SU}(6)$ wave functions. The form of the correction given in eq. (4) is an $O\left(p^{2}\right)$ approximation to the exact expression and while the shift applied to the $g_{A}$ above (after dividing out the 
neutron correction) is always less than $8 \%$, the individual corrections are much larger and the approximation is rather poor. The exact form of the correction for $A \rightarrow B \ell \nu$ may be written as

$$
\begin{aligned}
& g_{V}=g_{V}^{\mathrm{SU}(3)}\left[\epsilon_{A}^{+} \epsilon_{B}^{+}+\epsilon_{A}^{-} \epsilon_{B}^{-}\right] \\
& g_{A}=g_{A}^{\mathrm{SU}(3)}\left[\epsilon_{A}^{+} \epsilon_{B}^{+}-\frac{1}{3} \epsilon_{A}^{-} \epsilon_{B}^{-}\right],
\end{aligned}
$$

where $\epsilon_{i}^{ \pm}=\sqrt{\left(E_{i} \pm m_{i}\right) / 2 E_{i}}$, with $E_{i}=\sqrt{m_{i}^{2}+p^{2}}$. Again the correction to the vector coupling is never more than $0.2 \%$ and is thus negligible. Requiring that this reduce the $\mathrm{SU}(6)$ value of the neutron $g_{A}$ from $5 / 3$ to $\sim 5 / 4$, fixes $p^{2}=1.3 \mathrm{GeV}^{2}$ (which, a posteriori, demands use of the exact form of the expression). Choosing this value of $p^{2}$ and applying the exact formula we obtain the results shown in table $\mathrm{V}$.

Table V: An SU(3) breaking fit to the modified data including the external $V_{u d}$; the exact CoM correction is applied (see text for details).

\begin{tabular}{|c|c|c|c|c|c|}
\hline \multirow{2}{*}{ Data } & \multicolumn{3}{|c|}{ Parameters } & \multirow{2}{*}{$\chi^{2} /$ DoF } & \multirow{2}{*}{$F / D$} \\
\cline { 2 - 4 } & $V_{u d}$ & $F$ & $D$ & & \\
\hline$\Delta S=0$ & $0.9753 \pm 0.0007$ & $0.480 \pm 0.018$ & $0.785 \pm 0.018$ & 0.5 & 0.611 \\
\hline$|\Delta S|=1$ & $0.9747 \pm 0.0005$ & $0.464 \pm 0.009$ & $0.825 \pm 0.015$ & 1.0 & 0.563 \\
\hline All & $0.9744 \pm 0.0004$ & $0.460 \pm 0.006$ & $0.806 \pm 0.006$ & 1.0 & 0.570 \\
\hline
\end{tabular}

The unexpected result is a fit almost identical to the original approximate CoM correction with, however, the difference that here the choice of the parameter, $p^{2}$, was guided by the desire to explain the shift in the neutron $g_{A} / g_{V}$ from its $\mathrm{SU}(6)$ value; again it turns out to be very close to the best-fit value. Having said that, it is obvious that, although in this way we have "restored" the $\mathrm{SU}(6)$ picture for $F+D$, the individual $\mathrm{SU}(6)$ values of $F$ and $D$ are not recovered. Nonetheless, the $\Delta S=0$ decays do return a value still close to $F / D=2 / 3$.

To close this section, we remark on the possibility of including the type of wave-function mismatch correction mentioned above. In their bag-model calculation DHK arrived at an enhancement of the axial coupling by $8 \%$ due to this effect (while the vector coupling was reduced by 1.3\%). In the analysis performed here we have consistently found a preference for a small enhancement of both the axial and vector couplings, by about $2 \%$. However, within errors, the results are also consistent with zero effect. In other words, if both $V_{u d}$ and $V_{u s}$ are allowed to float a net tendency for over-saturation of CKM unitarity is observed (independently of whether or not external constraints on $V_{u d}$ are imposed). In all cases the ratio, $F / D$, is affected by at most $0.5 \%$. We note also that this explains the rather large value for $V_{u d}$ found in an earlier such analysis [4], which can thus be accounted for by a small renormalisation of the strangeness-changing couplings. 


\section{$5 \quad$ Recent approaches to $\mathrm{SU}(3)$ breaking}

Inspired by the observation that the $F / D$ ratios extracted from the angularcorrelation measurements display an approximately linear variation with the mass difference of the relevant hyperons with respect to the proton and neutron, Ehrnsperger and Schäfer [15] have attempted an SU(3)-breaking analysis and extraction of the $F$ and $D$ parameters. The idea is simply that the neutron $\beta$-decay $g_{A} / g_{V}$ provides the sum, $F+D$, and the remaining three known data values are used to make a one-parameter fit to an ad hoc correction:

$$
F / D=(F / D)^{\mathrm{SU}(3)}\left[1+a \frac{\left(m_{A}+m_{B}\right)-\left(m_{n}+m_{p}\right)}{\left(m_{A}+m_{B}\right)+\left(m_{n}+m_{p}\right)}\right],
$$

where $a$ is found to be $\sim 2.7$ and the limiting value of $F / D$ (valid for the nucleons) is $0.49 \pm 0.08$.

There are several criticisms to be levelled at such an approach, both of a formal theoretical nature and of a more practical type. First of all, let us recall that $F$ and $D$ are simply the antisymmetric and symmetric (in flavour indices) reduced matrix elements for charged-current baryon-baryon transitions. Thus, it is very hard to see why they should be renormalised in such a way as to miraculously maintain their sum constant while changing their ratio. Moreover, such a solution would imply that the decay $\Xi^{-} \rightarrow \Sigma^{0} \ell \bar{\nu}$ should have $g_{A} / g_{V}$ identical to that of the neutron, despite the enormous mass difference. Indeed, the breaking pattern so-predicted appears to be entirely random when viewed from the point-of-view of the various $g_{A} / g_{V}$. Put simply, there is an implicit, arbitrary and unexplained assumption in the ES approach: namely, that the particular combination $F+D$ is protected against $\mathrm{SU}(3)$ breaking.

On the practical side, the first and obvious objection is the neglect of the decay-rate data; we have repeated the ES analysis including all the data and find that the value of the breaking parameter, $a$, rises to $\sim 7$. While the overall $\chi^{2}$ is still admittedly very good, the assumption of linearity is now severely undermined and for certain decays $\left(\Xi^{-} \rightarrow \Lambda^{0} \ell \bar{\nu}\right)$ the effective renormalisation of $g_{A} / g_{V}$ is over $500 \%$. The second objection has to do with form chosen: it is a) linear in the mass breaking and b) neglects the mass difference between initial and final state (cf. the CoM correction). In the light of our findings and, e.g., the large $\Sigma^{ \pm}-n$ mass difference, neither assumption is justifiable or even plausible.

Moreover, as noted above, the angular-correlation data alone are well described even without SU(3) breaking. Thus, given the small lever-arm they offer (the only two precise data points lie very close in terms of the mass-breaking variable of ES), it is not surprising that this approach results in a very different value of $F / D$ with also very large errors. Note that, within the quoted errors, it does not significantly disagree with any of the other analyses

Let us now address the various approaches in which the validity of $\mathrm{SU}(3)$, and its use, is seriously questioned [2,6,22-24]. One of the main objections raised is 
the difficulty in explaining the baryon magnetic moments. While in no way wishing to embark on a discussion that would take us beyond the scope of this paper, we would counter such objections with two observations: firstly, the magnetic moments are exquisitely linked to the masses of the quarks, which (by definition) are unknown, while the axial couplings should not suffer such a complication. Second, a not entirely unsatisfactory picture of the magnetic moments can be obtained: the application of a similar correction to that used here allows a description that is good to within 0.07 nuclear magnetons over the whole octet (and also the $\Omega^{-}$decuplet resonance) [25]. It should always be borne in mind that a naïve two-parameter fit to the nucleon magnetic moments already reveals one of the important problems that makes this such a delicate subject: $-2 \mu_{d} / \mu_{u}=1.05$, indicating a $5 \%$ violation of isospin symmetry.

On the other hand, various of the proposals (see, e.g., $[23,24]$ ) do not imply a failure of the $\mathrm{SU}(3)$ description of the hyperon semi-leptonic decays but instead call into question the direct use of $F$ and $D$ in separating the quark spin contributions. However, the main thrust of the discussion presented here has been to demonstrate the applicability of SU(3) (and its known violations) to the extraction of the $F$ and $D$ parameters, without attempting to make any connection to the individual quark spins.

\section{Conclusions}

Before closing, let us try and identify those decays that could (with more precise measurement) throw useful light on this problem.

1. The $\Sigma^{ \pm} \rightarrow \Lambda^{0} \ell \nu$ decays are very significant. Firstly, they represent the major problem for an $\mathrm{SU}(3)$ symmetric fit and any significant increase in the measured rate for $\Sigma^{-} \rightarrow \Lambda^{0} e \bar{\nu}$ would greatly alleviate the situation. Second, as the only $\Delta S=0$ decays besides the neutron, they are valuable in helping to fix a reference point from which to judge the importance of corrections in the $|\Delta S|=1$ case.

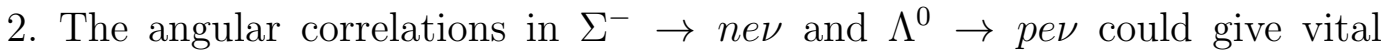
information on the importance of second-class currents, which in turn can quite dramatically affect the values of $g_{A}$ extracted and again seem to reduce the symmetry breaking necessary.

3. The $\Xi^{-} \rightarrow \Sigma^{0} e \nu$ rates and angular correlations, having the largest corrections, are also more sensitive, in principle, to any breaking. Moreover, the fact that $g_{A}=F+D$ for this process, identically to neutron $\beta$-decay, makes it uniquely interesting.

4. As a general observation, the angular correlations do not necessitate the 
inclusion of the CKM matrix elements in the fit and thus suffer less the ambiguity due to the, albeit small, discrepancy there.

The one blemish on the analysis presented here is that, despite motivating the corrections as being over an SU(6) symmetric "background", the values of $F$ and $D$ that finally emerge are still somewhat shifted. In mitigation of this failure we would remind the reader that the strangeness conserving data alone lie very close to the $\mathrm{SU}(6)$ picture. Moreover, inclusion of a possible correction for the strange-quark wave function does tend to raise the fit value of $F / D$. It was noticed in ref. [7] that the experimental data on the $\Sigma^{-} \rightarrow n e \bar{\nu}$ [10] decay also indicate a possible non-negligible second-class current contribution. Indeed, the data preferred a sizable $g_{2}$ and thus a much reduced value for $g_{A}(\sim 0.2)$. If this were the case then, firstly, the data would approach much more closely the $\mathrm{SU}(6)$ expectations (on inclusion of the corrections discussed here) and, secondly, the question arises of the relevance of such currents in other decays, where the experimental analysis has typically assumed them to be zero.

As for the implications in polarised DIS, the situation there is somewhat clouded by the inherent ambiguities (essential in PQCD and particularly due to the rôle of the anomaly) in defining separate quark-spin densities. The values of the parameters obtained here are very much in line with those generally used in the literature and so would not significantly alter the conclusions. Thus, the standard PQCD resolution of the Ellis-Jaffe sum rule discrepancy still demands some form of non-negligible strange quark polarisation.

We have hopefully convinced the reader that the use of SU(3) symmetry with well motivated corrections for its violation allows a very satisfactory description of the hyperon semi-leptonic decays and leaves little room for any further SU(3) breaking contributions. In support of the statement of validity we would remind the reader of the remarkable success of the fit motivated by the renormalisation of the neutron $g_{A}$ from $5 / 3$ to 5/4: in this approach no new free parameter was introduced and the initial $\chi^{2} / \mathrm{DoF}$ of over 2 was reduced to 1 . As far as other contributions or forms of correction are concerned, our analysis has shown that there is little space for radically different approaches when compared with the complete set of up-to-date experimental data. 


\section{References}

[1] J. Ashman et al., Phys. Lett. B206 (1988) 364; Nucl. Phys. B328 (1989) 1.

[2] H.J. Lipkin, Phys. Lett. B214 (1988) 429.

[3] F.E. Close and R.G. Roberts, Phys. Rev. Lett. 60 (1988) 1471.

[4] P.G. Ratcliffe, Phys. Lett. B242 (1990) 271.

[5] M. Roos, Phys. Lett. B246 (1990) 271.

[6] Z. Dziembowski and J. Franklin, J. of Phys. G 17 (1991) 213.

[7] F.E. Close, Rutherford preprint, RAL-93-034, hep-ph/9306288.

[8] J. Ellis and R. Jaffe, Phys. Rev. D9 (1974) 1444.

[9] M. Bourquin et al., Z. Phys. C12 (1982) 307; C21 (1983) 1; 17; 21.

[10] S.Y. Hsueh et al., Phys. Rev. D38 (1988) 2056.

[11] J. Dworkin et al., Phys. Rev. D41 (1990) 780.

[12] Particle Data Group, Montanet et al., Phys. Rev. D50 (1994) part VIII.

[13] SMC collab., B. Adeva et al., Phys. Lett. B302 (1993) 533; D. Adams et al., Phys. Lett. B329 (1994) 399.

[14] E143 collab., K. Abe et al., Phys. Rev. Lett. 74 (1995) 346; SLAC preprint, SLAC-PUB-95-6734.

[15] B. Ehrnsperger and A. Schäfer, Phys. Lett. B348 (1995) 619.

[16] For a recent review of the situation we refer the reader to I.S. Towner and J.C. Hardy, Chalk River preprint, nucl-th/9504015 (April 1995).

[17] I.S. Towner et al., Chalk River preprint, nucl-th/9507005 (July 1995).

[18] A. Garcia, R. Huerta and P. Kielanowski, Phys. Rev. D45 (1992) 879.

[19] P.N. Bogoliubov, Ann. Inst. Henri Poincaré 8 (1968) 163;

A detailed discussion of this type of correction may be found in: F.E. Close, An Introduction to Quarks and Partons (Academic Press, 1979).

[20] J. Donoghue, B. Holstein and S. Klimt, Phys. Rev. D35 (1987) 934.

[21] M. Ademollo and R. Gatto, Phys. Lett. 13 (1964) 264. 
[22] J. Franklin, Phys. Rev. D29 (1984) 2648.

[23] H.J. Lipkin, Phys. Lett. B337 (1994) 157.

[24] J. Lichtenstadt and H.J. Lipkin, Tel Aviv preprint, TAUP-2244-95.

[25] P.G. Ratcliffe, in preparation. 